\title{
INVESTIGATIONS OF ENGINE CHARACTERISTICS BY EXAUST GASES COMPOSITION CHANGE
}

\author{
Tamara Salova, Igor Ostyuchenko \\ Saint-Petersburg State Agrarian University, Russia \\ salova_tus@mail.ru
}

\begin{abstract}
For diagnostics of engines running, the operational characteristics are used, which are combined with the characteristics of the change in the exhaust gas composition. The implementation of driving cycles requires expensive equipment, the use of which demands significant costs. In the developed research methodology, it is proposed to use the simplification of the test system, replacing the driving cycles with equivalent test regimes, that is, from the acceleration modes to the equivalent modes that can be realized in bench tests.Each equivalent mode is calculated by the combination of two factors: the car speed and the power of resistance to vehicle movement. Values of concentration of harmful substances in exhaust gas at equivalent modes determine the level of car emissions. The composition of the exhaust gas at these modes, when compared with the calculated values of the components of the exhaust gas, makes it possible to select factors that affect the change in the course of the engine processes. In the developed model, the processes of mixture formation and combustion are considered as multi-phase processes of a multicomponent system. The process model is represented as the set of possible states of a real non-equilibrium process on the way to the final equilibrium state. The state of the final equilibrium of the combustion process is determined by the minimum value of the Gibbs function, when the material balance of the system is satisfied for the conditions $\mathrm{T}, \mathrm{P}=$ const. The developed model simplifies the procedure for calculating the composition of exhaust gas, since the calculation is based on the temperature of exhaust gas instead of the temperature of the combustion process. The methodology for calculating of exhaust gases composition can be realized for various heat engines; and allows to predict and carry out diagnostics of the engine fuel system.
\end{abstract}

Keywords: harmful emissions, exhaust gases, heat engines.

\section{Introduction}

The development of the theories of formation and distribution of emissions [1-6] showed that the most dangerous are spatially distributed pollution abatements, insignificant in number but having long-term actions, which include emissions with engine exitgases (ICE).

The main volume of harmful substances of ICE is thrown into the surrounding air together with the exit gases (EG). The most dangerous components of exit gases in transport operation are (in ascending order of toxicity degree): suspended substances, hydrocarbons, nitrogen dioxide and superecotoxicants, such as benz $(\alpha)$ pyrene. The degree of environmental pollution depends not only on the concentration of harmful substances in the exit gases of ICE, but also on the intensity of their release.

Long-term operation of ICE leads to a change in their operational parameters. The consequence of a violation in the fuel supply system is a reduction in the power and fuel efficiency of the engines under operating conditions, which affects the completeness of fuel combustion, the heat dissipation rate, and the toxicity of the exit gases. Therefore, the content of harmful components in the exit gases of the engine must be carried out taking into account the specific operating modes of the engine.

Thus, one of the ways to reduce environmental pollution during the operation of ICE is the development of methods and tools for internal combustion engines diagnosing.In modern ICEs with a microprocessor system, when using the scanning method, the knowledge of the exit gas composition greatly increases the probability of defect finding in the engine and notably shortens the time for engine systems troubleshooting. Being aware of the composition of the exit gas, you can make the necessary adjustments to the fuel supply system of the engine to obtain the optimum ratio engine power and fuel economy, make recommendations on engine repair need.

In connection with the foregoing, the development of a method for engine characteristics estimating by the exit gases composition in order to reduce the damage to the environment is of current interest.

To achieve this goal, it is necessary to solve the following objectives:

- to develop a methodology of engine toxicity assessment, when simulating operating modes in bench testing; 
- to develop a model and methodology for the ICE toxicity parameters calculating.

For diagnostics of engines running, the operational characteristics are used, which are combined with the characteristics of the change in the exhaust gas composition. In the conditions of transport operation, the engines most of the time work on unsteady modes, which make up the entire time of the movement:

- approximately $95 \%$ with intensive urban traffic;

- about $85-90 \%$ when driving on dirt roads;

- $30-35 \%$ on suburban improved roads.

Therefore, for the internal combustion engine toxicity assessment, driving cycles are used: simple urban and out-of-town, consisting of idle run, acceleration, steady-state motion, deceleration and braking [7]. The implementation of driving cycles requires expensive equipment, the use of which demands significant costs. In the developed research methodology, it is proposed to use the simplification of the test system, replacing the driving cycles with equivalent test regimes [8], that is, from the acceleration modes to the equivalent modes that can be realized in bench tests. The number of equivalent modes is determined based on the analysis of the driving cycle schedule. The stages of acceleration are represented by points, the number of which is equal to the number of gear box stages used during acceleration; stages of uniform motion are represented by a single point, the stages of idle run and deceleration are represented by single point of idle run. Thus, the urban driving cycle is represented by 11 regimes (Table 1 ).

Table 1

Equivalent tests of urban driving cycle

\begin{tabular}{|c|c|c|c|c|}
\hline Stage name & Speed, $\mathbf{~ k m} \cdot \mathbf{h}^{-\mathbf{1}}$ & Speed-up, $\mathbf{~ m \cdot \mathbf { s } ^ { - \mathbf { 2 } }}$ & Time, $\mathbf{s}$ & Step \\
\hline 1 acceleration & $0-15$ & 1.04 & 4 & 1 \\
\hline 2 constantspeed & 15 & - & 8 & 1 \\
\hline 3 acceleration & $0-15$ & 0.83 & $5+5=10$ & 1 \\
\hline 4 acceleration & $15-32$ & 0.94 & 5 & 2 \\
\hline 5 constantspeed & 32 & - & 24 & 2 \\
\hline 6 acceleration & $15-35$ & 0.62 & 9 & 2 \\
\hline 7 acceleration & $35-50$ & 0.52 & 8 & 3 \\
\hline 8 constantspeed & 50 & - & 12 & 3 \\
\hline 9 constantspeed & 35 & - & 13 & 3 \\
\hline 10 idle run & - & - & 77 & - \\
\hline 11 idle run & - & - & 25 & - \\
\hline
\end{tabular}

Each equivalent mode is calculated by the combination of two factors: the car speed and the power of resistance to vehicle movement. The car speed is determined by the engine rotation speed, the power of the resistance to movement is determined by the stand load.

\section{Materials and methods}

For the research, the wide spread in Russia automobile (GAZ 3110) engine ZMZ-4062 of the Zavolzhsky Engine Plant had been chosen. We determined the parameters of the engine operation at equivalent modes shown in (Table 2). Modes of the engine work are conventionally divided into three areas: small loads, medium loads, heavy loads, to reduce the number of research modes. All points that fall into one zone are represented as one, and after grouping, there are only 4 equivalent modes located in all areas of the engine [9]. Values of concentration of harmful substances in exhaust gas at equivalent modes determine the level of car emissions. The composition of the exhaust gas at these modes, when compared with the calculated values of the components of the exit gas, makes it possible to select factors that affect the change in the course of the engine processes. 
Engine power and economy power change connected with change processes of fuel formation and fuel combustion are interrelated with changes in pressure and temperature in the engine cylinder. Therefore, the analysis of the change in the thermodynamic potential or the Gibbs function is based on the model and methodology for calculating the toxicity parameters of the internal combustion engine. The value of the Gibbs function with known parameters of the pressure combustion process and temperature is determined from the change in the composition of the fuel-air mixture.

Table 2

Working parameters of engine ZMZ-4062.10 on equivalent tests

\begin{tabular}{|c|c|c|c|c|c|}
\hline $\begin{array}{c}\text { No. of } \\
\text { regime }\end{array}$ & Power, $\boldsymbol{N}_{\boldsymbol{i} \boldsymbol{k}}$ & $\begin{array}{c}\text { Revolution } \\
\text { rate } \boldsymbol{n}_{\boldsymbol{i}}\end{array}$ & $\begin{array}{c}\text { Rotation } \\
\text { torque } \boldsymbol{M}_{\boldsymbol{i} \boldsymbol{e}}\end{array}$ & $\begin{array}{c}\text { Weight } \\
\text { coefficient } \boldsymbol{k}_{\boldsymbol{i}}\end{array}$ & Range of loads \\
\hline 1 & 0.646 & 1768 & 70 & 0.0125 & High \\
\hline 2 & 2.153 & 2481 & 80 & 0.0225 & Low \\
\hline 3 & 3.660 & 2500 & 113 & 0.0200 & Medium \\
\hline 4 & 5.166 & 2624 & 143 & 0.0650 & High \\
\hline 5 & 6.028 & 2505 & 94 & 0.1250 & Low \\
\hline 6 & 4.305 & 2354 & 55 & 0.1725 & Medium \\
\hline 7 & 5.166 & 2505 & 88 & 0.1250 & High \\
\hline 8 & 7.319 & 2827 & 174 & 0.0875 & Low \\
\hline 9 & 8.611 & 3150 & 148 & 0.0750 & Low \\
\hline 10 & 9.472 & 3365 & 194 & 0.0500 & Idle run \\
\hline 11 & 10.333 & 3580 & 181 & 0.0250 & Idle run \\
\hline
\end{tabular}

In the developed model, the processes of mixture formation and combustion are considered as multi-phase processes of a multicomponent system. The process model is represented as the set of possible states of a real non-equilibrium process on the way to the final equilibrium state (Fig. 1). The state of the final equilibrium of the combustion process is determined by the minimum value of the Gibbs function, when the material balance of the system is satisfied for the conditions $\mathrm{T}, \mathrm{P}=$ const:

$$
\min \left[\Phi(x)=\sum_{j} \Phi_{j}(x) \cdot x_{j}\right],
$$

on condition

$$
A \cdot x=b, x_{j} \geq 0,
$$

where $\Phi-$ value of the Gibbs function;

$A=\left[a_{i j}\right]-$ matrix of $\mathrm{m} \times \mathrm{n}$ contents of elements in the components of the system;

$x=\left(x_{1}, \ldots x_{n}\right)-$ vector of the molar content of the components;

$b=\left(b_{1}, \ldots b_{m}\right)-$ vector of the number of moles of elements.

The obtained regularities make it possible to give a complete thermodynamic characteristic of the combustion processes of fuel, including a qualitative and quantitative change in the composition of the fuel-air mixture, depending on the combustion conditions.

The model is translated into an algorithmic language and implemented as a software package [10]. In the software package it is taken into account that the thermodynamic model allows to investigate the influence of various factors: temperature and pressure, the amount of oxygen in the gas-air mixture, the composition of fuel for the mixture formation process and the final combustion products formation. The model makes it possible to estimate the composition of the exit gas at various operating conditions of the engine. 


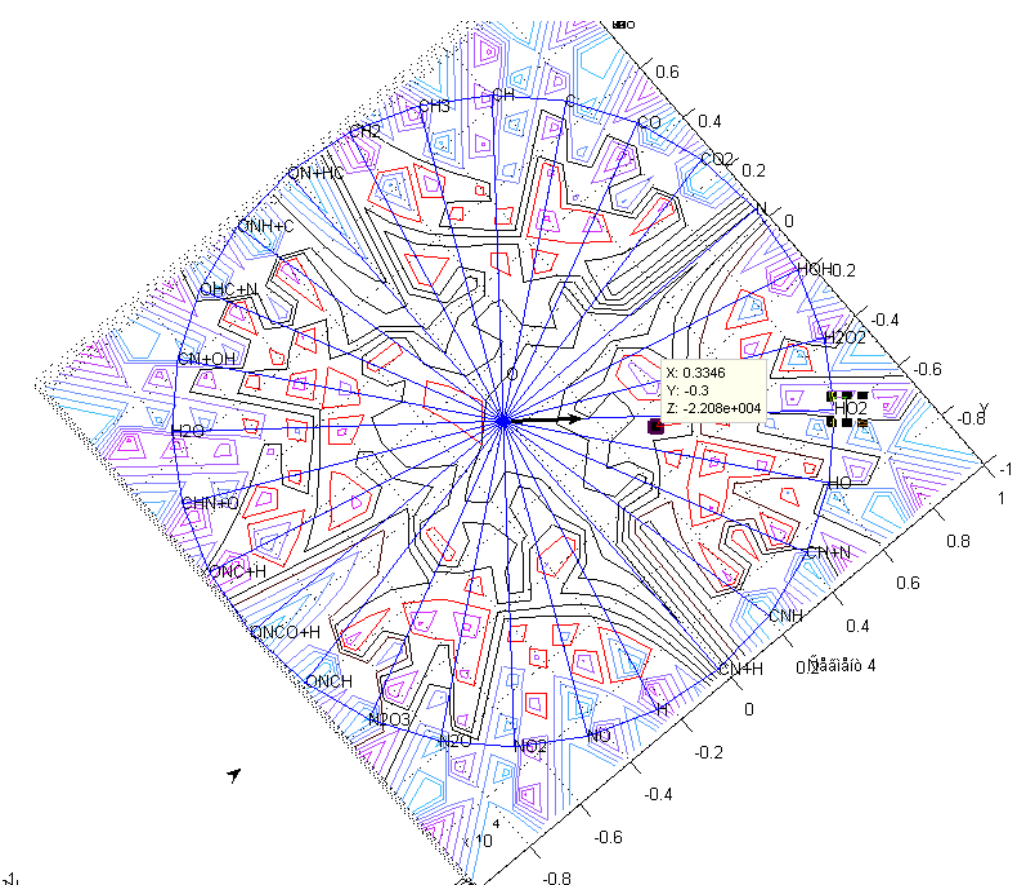

Fig. 1. Thermodynamic model of combustion process: material balance of the system; value of the Gibbs function $\Phi$ (black and red lines); - minimum value of the Gibbs function (a red line)

\section{Results and discussion}

To find the direction of decreasing the Gibbs function, calculations of the objective function are made when changing the variables-the composition of the system (equations 1,2). Next, using the gradient of the Gibbs function, we determine the local minimum of the value of the Gibbs function, which corresponds to the equilibrium state of the system.

Estimation of the exit gas composition for the obtained equilibrium state of the system was carried out using the developed software package.

To determine the value of the Gibbs function, it is necessary to know the parameters of the working process-the pressure and temperature of the combustion process, which are determined by the results of the combustion engine identification (Fig. 2), which significantly complicates the model. To simplify the model, an analysis was made of the dependence of the change in the temperature of the combustion process and the temperature of the exit gas for the regimes typical for engine operating conditions.

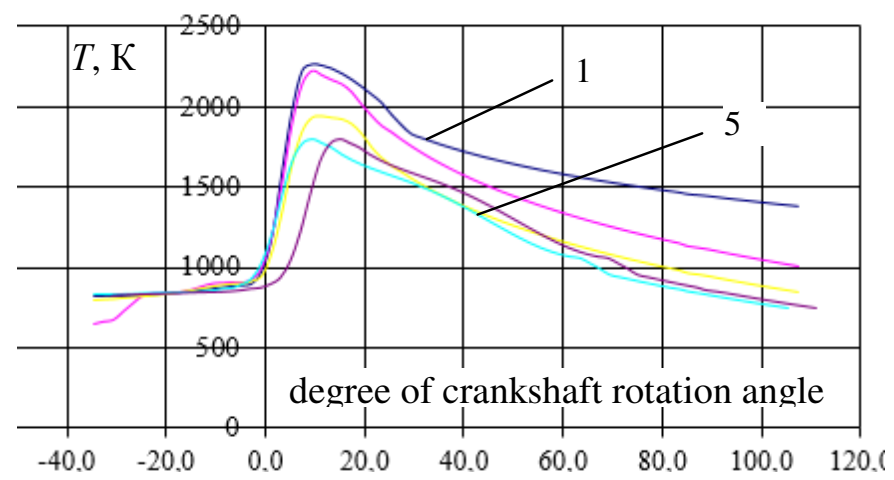

Fig. 2. Temperature (T) changing of combustion engine ZMZ-4062.10,depending on crankshaft rotation angel for operating modes in range from rated power to maximum torque:

$$
1-5-\text { load } P e=90,80,70,60,50 \% \text { maximum }
$$

Analysis of the dependencies showed that the change in the combustion temperature of the fuel can be determined from the temperature of the exit gases of the $T_{e g}$ (Figs. 3, 4), using thedependence obtained 


$$
k=-0.0006 x^{3}-0.03 x^{2}+0.587 x+0.965 T_{c}=k T_{e g},
$$

where $x$-dimensionless value of the load, when the load varies from 0 to 10 .

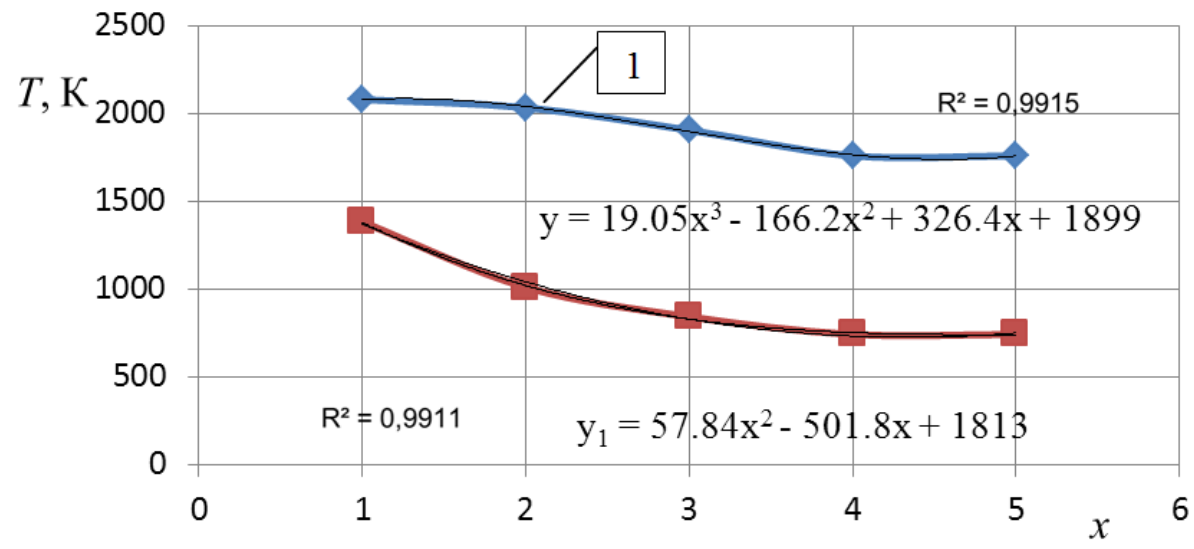

Fig. 3. Temperatures changing of combustion process (1) and exhaust gases of engine from load $x$

According to the developed methodology, the characteristics of equivalent modes that simulate the conditions of transport operation are determined, which allow to define the emissions toxicity in bench conditions. Knowing emissions of exit gas toxic components reduces the time to troubleshoot the engine systems.

The developed thermodynamic model and the procedure for calculating the process of fuel combustion makes it possible to establish the equilibrium state of the system under given conditions of mixture formation and combustion, which determines the composition of the exit gas.

The developed model simplifies the procedure for calculating the composition of exit gas, since the calculation is based on the temperature of exit gas instead of the temperature of the combustion process.

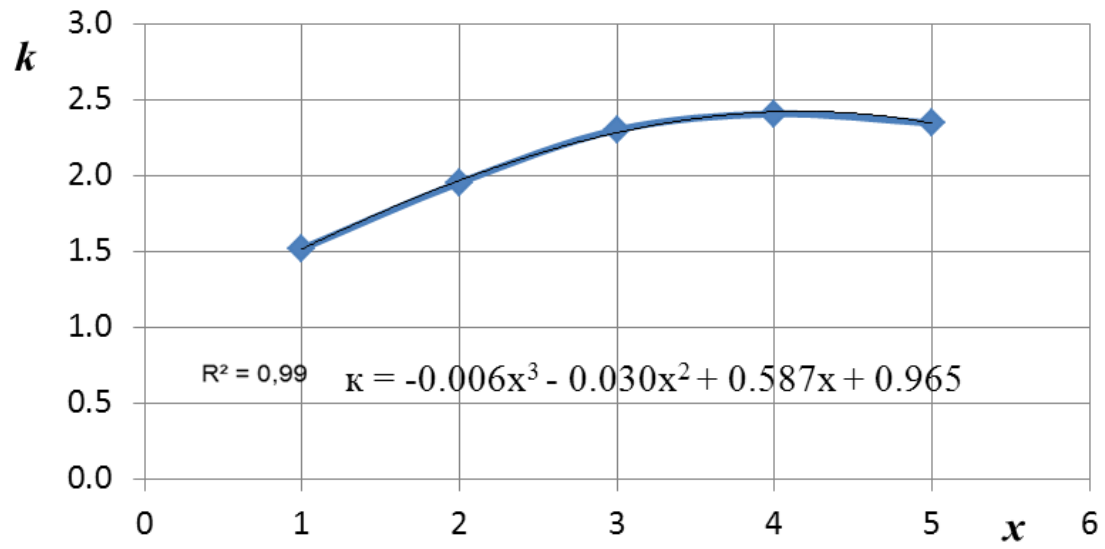

Fig. 4. Dependence of dimensionless coefficient $\mathrm{k}$ on load value $x$

\section{Conclusions}

1. The proposed procedure allows to determine the values of components concentration in the exit gases, including those on modes equivalent to unsteady modes, specific for the movement of mobile machinery.

2. The methodology for calculating of exit gas composition can be realized for various heat engines; it allows to predict and carry out diagnostics of the engine fuel system.

3. The implementation of the methodology for heat engines diagnosing on the exit gases composition can reduce the emission of toxic substances by operating conditions of mobile machinery. 


\section{References}

[1] Antropov I.V., Kronrod B.A. On the dependence of the evolution of a term in a stratified medium on the initial conditions - Izv. AN SSSR. Physics of the Atmosphere and the Ocean - Vol. 25 No. 12 - 1989, pp. 1261-1266.

[2] Fick A. Veber diffusion - Ann. Phys. u. Che-mie. (J.C. Poggendorff) - 1855 - 94(170), pp. 59-86.

[3] GOST R 41.83 - 2004 Uniform instructions concerning the certification of vehicles with respect to harmful substances emissions depending on the fuel required for engines.

[4] Morton B.R., Taylor G.Y., Turner J.S. Turbulent gravitational convection from maintained and instantaneous sources.- Proc.Royal Soc., London,1956. A234, №1196, pp. 1-23.

[5] Ogura Y. Convection of isolated masses of buoyant fluid: a numerical colculation - J. Atm. Sci. v. 19 - № 6 - 1962

[6] Rouse H., Jih C.-S., Humphreys H.W. Gravitational convection from a boundary source. Tel-lus, 1952, v.4, pp.201-210.

[7] Richards J.M. The effect of wind shear on puff.- Quart. J. R. Met. Soc. - 96 - 1970, pp. 702-714.

[8] Salova T.Yu., Korabelnikov S.K. Assessment of harmful emissions formation process of diesel engines / Bulletin of the Academy of Military Sciences. Moscow. № 1 (30) 2010, (special issue) pp. 80-87.

[9] Salova T.Yu. Sivov A.A. Investigation of work indexes of the nitrogen oxides neutralizer in gasoline engine while using the cars in urban conditions // Izvestiya of International Academy of Agrarian Education. SPb .: SPbSAU № 13 (volume 2), 2012, pp. 76-81.

[10] Salova T.U., Korabelnikov S.K. The calculation of combustion materials 1.0. Official accreditation certificate of profile for ECM №2006612767. Reg. 04.08.2006. 